\title{
Study on Medical Treatment of Injury in Football
}

\author{
${ }^{1}$ Shanguo Liu, ${ }^{2}$ Yue Wu \\ ${ }^{1}$ Department of Sports Rehabilitation and Physical Education, Changsha \\ Medical University, Changsha, 410219, China \\ ${ }^{2}$ The First Affiliated Hospital of Changsha Medical University, Changsha, \\ 410219, China
}

\begin{abstract}
In this paper, we make the investigation on the common sports injuries, the type of injury in college students. We use the knowledge of sports anatomy of common sports injuries to analyze the prevention of college football students' injury. The healthy development of football has a certain practical significance on the college football educators for safety education. It also has certain theoretical significance prevention on football injury and injury treatment.
\end{abstract}

Keywords: study, medical treatment, injury, football

\section{Introduction}

The development of football has been a hundred years of history, and more and more people like the masses, like to watch the ball more and more people play, young people are increasingly obsessed with football. Because of the encouragement and confrontation of sports, athletes often suffer from injury. The occurrence of various injuries is often restricted by the athletes' participation in the sports and has close relationship with the technical characteristics of the project and the physiological characteristics of the human body. Modern competitive sports is full of fierce competition, the strength of physical confrontation between players is becoming increasingly fierce, which also makes a lot of athletes will be some damage. The coaches, athletes and medical and scientific research staff of sports participating are responsible for helping the 
athletes to improve the level of self-competition. The athletes rely on the coaches to improve the self-physical ability and the level of technical and tactical. The researchers and medical personnel for the athlete's technical planning and nutrition and health care to do a detailed plan, multi-efforts are for the athletes to create a more excellent race results. Therefore, athletes only continue to challenge their own physical limit is possible to complete the breakthrough results, and this breakthrough occurs when the athletes injury.

In China, the development of football programs is very popular. The students participating in the activities are also increasing year by year. Because of its strong antagonism, fast speed, fierce competition and strong strength, the athletes are often injured. As some coaches athletes training is unscientific and reasonable, the probability of athletes injury is also increasing, always plaguing coaches and athletes, restricting the level of our college football program to further improve. According to statistics $\backslash$ In recent years, college students in China's participation in football sports injuries are also increasing the chance, on the one hand is due to the increase in the number of football projects; the other hand, mainly because of students to The mechanism of sports injury do not understand, how to avoid sports injury is not clear. In the event of injury, it will naturally affect the healthy growth of student athletes, shorten their sports life; at the same time, will also affect the cultural studies.

\section{The common injuries of football}

Football is very confrontational, players in the game can use a variety of running, emergency stop, turn around, fell to the ground, jumping, collision and other actions. This feature of soccer determines that football is one of the most vulnerable sports, but in addition to high-level competition with team doctors, the general grass-roots competition does not have this condition, so the need for grass-roots coaches and players To have this limited conditions of first-aid ability, the following are some of the most common football injury first aid methods to make some introduction.

\subsection{Fracture}

Most parts of the fracture in the calf of the tibia and fibula, usually caused by direct violence of the closed fracture, the pain was lighter in the fracture, but the activities of limbs, the pain increased, because the tibia before the soft tissue is thin, so Generally visible hematoma infiltration subcutaneous, the formation of purple subcutaneous, fracture can not stand, walking, severe fibular fractures can also be found after the deformation of the body, in short, light fractures without obvious systemic symptoms, but severe shock-prone.

If the athletes have signs of leg fractures, first let the patient limb in a static state, and then immediately find a fixed material; two width appropriate (5 $10 \mathrm{~cm}$ ) of the wood, the length of the leg longer than the wood, Bamboo can also be replaced, cloth or rope 4 . Materials ready, the splint were placed inside and 
outside the calf on both sides, up to the lower thigh, the foot was issued under the foot, with the cloth were in the knee, knee and ankle tied tied fixed, and then tie the leg and leg health tied Together immediately after the hospital treatment.

\subsection{Soft tissue injury}

Grass-roots football, because of the constraints of space and clothing conditions, lying in the action occurred in the probability of abrasion is almost $100 \%$, but the severity of the points. Wounded, the smaller the area of abrasions, can be cleaned with saline after local rub to red mercury or violet syrup, no bandage, but the joint parts of the abrasions coated with antibacterial ointment as much as possible after the dressing, the more serious abrasions in general Buttocks and knees at the bottom, the wound is also often cinder and sand and other foreign matter, washed with saline can be used when the toothbrush disinfected, and with hydrogen peroxide, wound around $75 \%$ alcohol disinfection and dressing.

In the football game, closed soft tissue injury is the most and most common, this damage the skin without cracks, often after injury tissue bleeding, tissue fluid exudation, the formation of local hematoma or congestion, usually contusion, sprain, strain, If the treatment is not timely or inappropriate, not only will extend the course of treatment, may also affect the future in football.

In the course of football in the event of closed soft tissue injury, should be based on the specific circumstances of injury to make timely and appropriate treatment, first of all to follow the brake, anti-swelling, analgesic basic principle, then the injury site still Ice, etc., such as the scene without cold water or ice, can also be directly used to bandage cloth, such as the scene without bandages, cloth, can be directly crushed by hand or around the office of the aorta, to prevent injury site bleeding, the emergence of swelling, in particular, should be noted that this time do not massage the Ministry of injury, which not only can not restore the injury, but also increased tissue bleeding and tissue fluid exudation, swelling Increase. After completing the simple treatment, should immediately go to the hospital for X-ray examination.

\section{The injury medical treatment methods in football}

\subsection{Cold method}

The immediate use of the cold method for athletes injured on the field is essential. Methane sprays are essential basic treatment equipment for every trainer. But this method of muscle sprain, dislocation and muscle injury and other symptoms can only play a role in reducing pain. Therefore, once the hematoma phenomenon, absolutely can not spray methane, but must use the ice bag swelling method.. This ice bag should be replaced once every $10 \mathrm{~min}$, cold time at least $1 \mathrm{~h}$. In the treatment of hematoma .Do not use the skin heating agent or alcohol swelling, because this treatment will enlarge the area of congestion, increased internal 
bleeding .Although ice cream hematoma for a long time, but because the wound is always $0^{\circ} \mathrm{C}$ state, so the effect of very good.

\subsection{Pozzolana therapy}

After 24-48 hours of cold therapy, the temperature of the hematoma area is close to the normal body temperature, and the subcutaneous tissue congestion, injury, and hematoma can be treated with hyperthermia. The paraffin is heated to a porridge-like shape and then stirred with the volcano to continue heating to $50^{\circ}$ $\mathrm{C}-60^{\circ} \mathrm{C}$ and is loaded into a plastic bag and placed in the wound. This therapy by the athletes themselves can be carried out.

\subsection{Massage therapy}

Massage is the main means of kneading, kneading, hammering, grinding and massage. This ancient tradition of treatment should generally be experienced masseurs to carry out, because they can be based on the athlete's injury time and location using accurate some old players can even self-massage, this can speed up the recovery rate .In addition, there is a small massage therapy.

\subsection{Special therapy}

Physiotherapy for the treatment of athletes' injuries provides a variety of means, one of the important therapies is the traditional Chinese Cupping therapy. This treatment of rheumatism, back pain, muscle crush, headache, abdominal pain and other curative effect is very good. Cupping suction to help drainage or drainage and it is a simple and effective treatment.

\subsection{Exercise therapy}

After the above treatment, the injured part of the athletes returned to normal activities and then it should begin active and active self-motion therapy, such as swimming, cycling, jogging and exercise, etc. Of course, the early athletes during the exercise otherwise, it will cause further deterioration of the injured muscle tissue, increase the bleeding area, so that has already begun to produce metabolic changes in the muscle cell counter-encirclement, muscle tissue will harden or stop the formation of new tissue.

\subsection{The treatment of muscle strain}

Due to pre-game preparation activities is not sufficient or the original injury site has not been completely eradicated in the game, a lot of sweat caused by excessive salt loss in the body, can easily cause muscle strain. The symptoms most likely to occur in athletes sudden burst of explosive force running Movement, the direction of movement changes, strong strokes between the road and fast running in a sudden pause. At this time, due to the elasticity of the muscle beam over the limit and excessive elongation, resulting in muscle internal 
hyperemia, if not treated, it will produce muscle tear. When this happens, the athlete must immediately stop the game, by the doctor to take massage to relieve muscle tissue spasticity, and then tied in the wound splint or bandage. If the pain intensified, the athletes should be immediately carried out of the field, should immediately use Cold treatment $.2 \mathrm{~h}$, the use of hot bandage wrapped around the affected area $.24 \mathrm{~h}$, to be pain relief, you can use massage or ultrasound to continue treatment.

\subsection{The treatment of muscle tear}

Muscle strain, such as continue to increase after the amount of exercise, it is easy to develop into muscle tear. At this time, the muscles will produce needle-like pain, resulting in loss of mobility of injured parts, which can be judged as muscle tearing its symptoms as the injured parts of the muscle tissue inelastic and weak. Visual impression is that after a few hours there will be subcutaneous sag, which shows that muscle tear often can cause severe hematoma .Then appear muscle tear, you must immediately take the cold method, the need to wrap a bandage. After $24 \mathrm{~h}$, should be as much as possible every day for ice massage or each time I0min cold water massage bath and ultrasonic therapy, 3-4 days later, injured athletes can be the right amount of relaxation training. A week later, you can jog, if there is no feeling of discomfort, you can begin to participate in training.

\section{Conclusion}

With the increasing concern of football management, athletes and coaches increased the attention degree of sports injuries, the quality of scientific research personnel has improve, the domestic football players will greatly reduce the risk of injury, Chinese football will get healthy and smooth development.

\section{References}

[1] Ma Yuhong. Research on the Sports Injury of the Students Majoring in Physical Education. Liaoning Sport Science and Technology, 55(12), pp.68-70, 2011

[2] Lu Wenyi. An Analysis of Students' Soccer Injury. Journal of Sichuan Sports Science, 8 (5), pp.87- 91, 2013

[3] Du Yongjun. Football teaching and training in the injury and prevention. Chinese school sports, 12(10), pp. 18- 20, 2012

[4] Shiquan Quan. Investigation and Analysis of Football Injuries in Colleges and Universities, Journal of Beijing Sport University, 9(6), pp.58- 61, 2011

[5] Wang Xinguan. Investigation and analysis of the common trauma of college students' football, Journal of Wuhan Institute of Physical Education, 8(4), pp.8587,2014 